\title{
烷基取代的 1,3-二烯区域可控还原烯基化反应
}

\author{
陶相华 $a$ 龚和贵*,a,b \\ ( ${ }^{a}$ 上海大学材料科学与工程学院 上海 200444) \\ ${ }^{b}$ 上海大学化学系 超分子化学与催化中心 上海 200444)
}

\begin{abstract}
Regiocontrolled Reductive Vinylation of Aliphatic 1,3-Dienes
Tao, Xianghua ${ }^{a} \quad$ Gong, Hegui ${ }^{*}, a, b$

( ${ }^{a}$ School of Materials Science and Engineering, Shanghai University, Shanghai 200444)

( ${ }^{b}$ Center for Supramolecular Chemistry and Catalysis, Department of Chemistry, Shanghai University, Shanghai 200444)
\end{abstract}

过渡金属催化 1,3 -二烯选择性可控的氢一碳化反应 在有机合成中具有很高的研究价值 ${ }^{[1]}$. 通常情况下, 此 类反应会经历 $\eta^{3}$ - $\pi$-烯丙基金属中间体，从而得到相应 的 1,2-和 1,4-加合物. 此外, 由于上述中间体在反应过 程中处于平衡状态, 导致反应中往往伴随着顺反异构化 的现象(Scheme 1-1). 因此, 区域选择性的调控是二烯 化学领域中颇具挑战性的研究课题. 以往的研究表明: 1,3-二烯氢化-官能团化反应的选择性与二烯类底物的 电性和空间结构密切相关. 因此, 迄今为止绝大多数已
报道的选择性可控反应主要集中于芳香族 1,3-二烯类化 合物. 其中, 二烯与亲核试剂反应得到 branch 选择性的 1,2-加合物 ${ }^{[1-2]}$ (Scheme 1, 2a); 二烯与亲电试剂反应得到 Linear 构型的产物 (Scheme 1, 2b $)^{[3-4]}$. 相比之下, 脂肪族 1,3-二烯的区域选择性调控一直未能得以解决. 目前的 研究方案主要是采用具有环系结构的二烯 ${ }^{[5]}$ 和大位阻的 底物 ${ }^{[3,6-7]}$. 近日, 兰州大学化学化工学院舒兴中课题组 在该领域取得了突破性的进展. 他们发展了一类膦-氧 基类配体，该配体可与 $\mathrm{Ni}(0)$ 作用形成四聚络合物
Challenge in the field

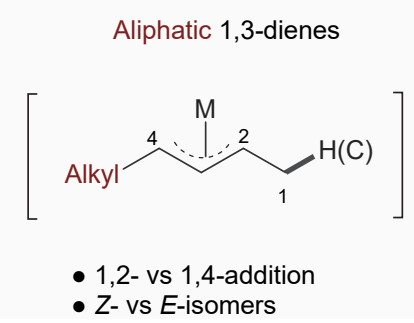

2 Selectivity profiles of 1,2-hydrocarbonation

(a) Reactions with nucleophiles (many reports)

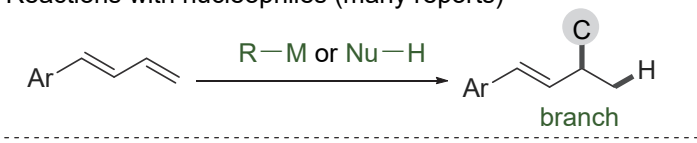

(b) Reactions with electrophiles (rare)

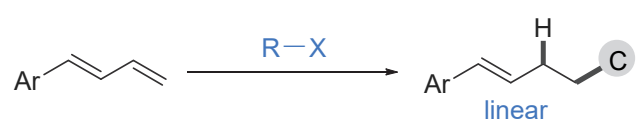

3 Branch-selective 1,2-hydrovinylation of 1,3-dienes with vinyl triflates (this work)
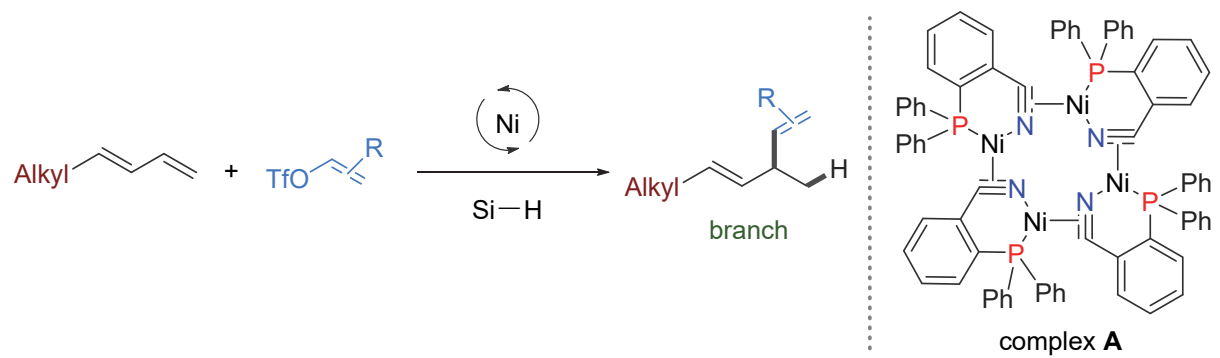

图式 1 1,3-二烯的 1,2-氢-碳化反应

Scheme 1 1,2-Hydrocarbon functionalization of 1,3-dienes

*Corresponding author. E-mail: hegui_gong@shu.edu.cn. Published online April 22, 2021. 
(complex A). 该络合物被证实具有特殊的催化活性, 可 以规避经典的 $\eta^{3}-\pi$-烯丙基金属中间体历程，从而有效 解决了普通脂肪族二烯的反应选择性问题 (Scheme 1, 3). 除此之外, 芳香族 1,3-二烯、丁二烯以及共轭多烯均 能以优异的选择性得到 branch 类型 1,2-加成产物.

作者前期对配体进行了考察研究, 烯烃还原氢化官能团化反应中常用的含氮配体对反应的转化以及选 择性控制几乎无效; 使用常见的膦配体时反应也不能进 行. 后来, 作者发现膦一氰基类配体能够作用于该反应. 通过后续对此类配体的电性以及位阻进行调整, 反应最 终能够区域可控地实现脂肪族二烯的高效转化.
在得到最优条件后，作者对反应的普适性进行了考 察(Scheme 2). 实验结果表明，该反应具有广泛的底物 适用范围，能够高效地实现小位阻脂肪族二烯、共轭多 烯以及丁二烯的选择性调控, 以优异的化学、区域、立 体选择性得到相应的目标产物. 另外, 富电子、电中性 以及缺电子芳香族二烯也均适用于该反应体系. 对于烯 基磺酸酯，该反应也体现出了良好的适应性. 一系列环 系和非环系的烯基磺酸酯均能高效地转化为相应的多 烯化合物. 此外，该反应具有良好的官能团兼容性，能 兼容硅醚、羟基、缩酮、酯基、杂环以及酰胺等官能团. 该反应可实现克级规模的制备，同时适用于诺卡酮、睪

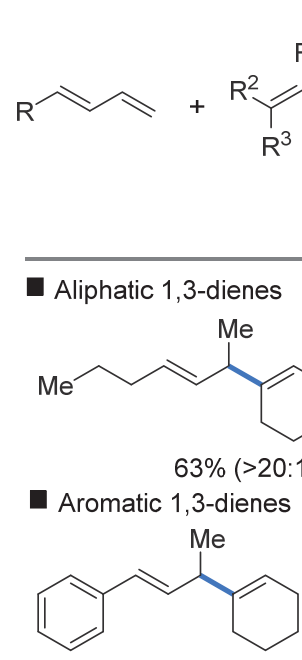

$72 \%(>20: 1)$

- Aromatic 1,3-dienes

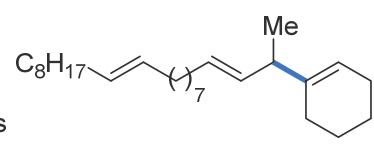

$66 \%(>20: 1)$
L (6 mol\%) PMHS (2.5 equiv.)

THF/DIPE ( $V: V=3: 1,0.2 \mathrm{~mol} / \mathrm{L})$ $\mathrm{CsF}$ (2.0 equiv), $0^{\circ} \mathrm{C}$

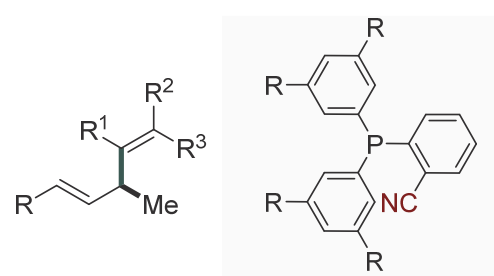

$\mathbf{L}(\mathrm{R}=\mathrm{H}$ or $t-\mathrm{Bu})$

The substrate scope of 1,3-dienes<smiles>CCOC(C(=O)OC=CC(C)C1=CC[NH+](C)CC1)c1ccccc1</smiles>

$82 \%(>20: 1)$

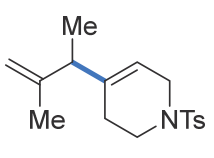

$51 \%(>20: 1)$

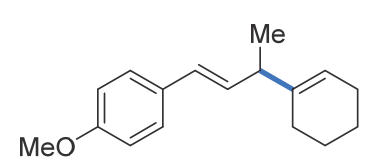

$91 \%(>20: 1)$<smiles>CC(=O)c1ccc(/C=C/C(C)C2=CCCCC2)cc1</smiles>

$92 \%(>20: 1)$

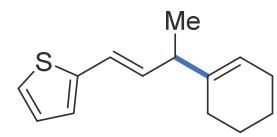

$70 \%(>20: 1)$

The substrate scope of vinyl triflates
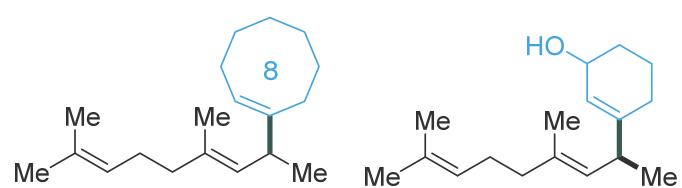<smiles>[M]C(/C=C(\C)CCC=C(C)C)C1=CCN([Tl])CC1</smiles><smiles>CC(C)=CCC/C(C)=C/C(C)C1=CCOCC1</smiles>

$69 \%(>20: 1)$

$60 \%(>20: 1)$

$85 \%(>20: 1)$

$1.3 \mathrm{~g}, 84 \%(>20: 1)$

$81 \%(>20: 1)$<smiles>C=C(CCCCC(=O)Nc1ccc(C)cc1)C(C)C=C(C)CCC=C(C)C</smiles>

$80 \%(>20: 1)$<smiles>C=C(CCCCCOC(=O)C(C)c1ccc(-c2ccccc2)c(F)c1)C(C)C=C(C)CC</smiles>

$74 \%(>20: 1)$
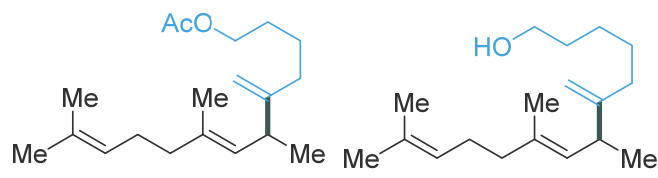

Modification of biologically active molecules
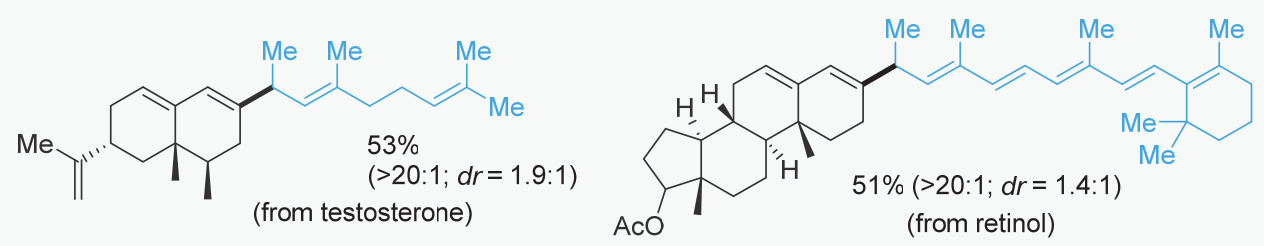

图式 2 反应的普适性及应用性考察

Scheme 2 Scope of the substrates and applications 
酮和维生素 $\mathrm{A}$ 等一系列复杂生物活性分子的后期修饰.

机理研究实验表明, 该反应的催化机制不同于常见 的氢化一官能团化反应, 避免了经历 $\eta^{3}-\pi$-烯丙基金属中 间体过程. 具体催化过程如 Scheme 3 所示: Ni(0)首先与 vinyl-OTf 发生氧化加成反应生成 vinyl-Ni(II)-OTf 中间 体, 之后该络合物与 $\mathrm{Si}-\mathrm{H}$ 发生金属交换得到 vinyl$\mathrm{Ni}(\mathrm{II})-\mathrm{H}$; 该中间体与二烯进行 vinyl-Ni(II)选择性插入 反应和还原消除得到目标产物.

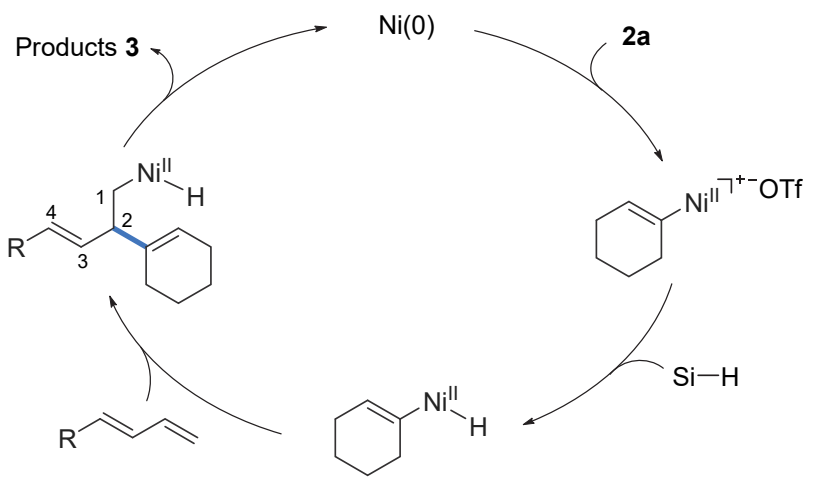

图式 3 可能的反应机理

Scheme 3 Possible reaction mechanism
综上所述, 舒兴中课题组发展了一种新型的催化体 系，为 1,3-二烯的 branch 选择性 1,2-加成反应提供了一 种较为普适的解决方案. 反应代表了该领域一类崭新的 选择性加成模式，适用于脂肪族、芳香族 1,3-二烯以及 共轭多烯的还原烯基化反应，为 1,4-二烯的合成提供了 一条简洁高效的途径.

\section{References}

[1] Li, G.; Huo, X.; Jiang, X.; Zhang, W. Chem. Soc. Rev. 2020, 49, 2060.

[2] Adamson, N. J.; Malcolmson, S. J. ACS Catal. 2020, 10, 1060.

[3] Saini, V.; O'Dair, M.; Sigman, M. S. J. Am. Chem. Soc. 2015, 137, 608.

[4] Lv, L.; Yu, L.; Qiu, Z.; Li, C.-J. Angew. Chem., Int. Ed. 2020, 59, 6466.

[5] RajanBabu T. V.; Adam Cox G.; Lim H. J.; Nomura N.; Sharma R. K.; Smith C. R.; Zhang, A. In Organic Synthesis Comprehensive Organic Synthesis, Vol. 5, 2nd ed., Oxford, Elsevier, 2014, pp. $1582 \sim 1620$

[6] Liao, L.; Sigman, M. S. J. Am. Chem. Soc. 2010, 132, 10209.

[7] Xiao, L. J.; Cheng, L.; Feng, W. M.; Li, M. L.; Xie, J. H.; Zhou, Q. L. Angew. Chem., Int. Ed. 2018, 57, 461.

[8] Pang, X.; Zhao, Z.-Z.; Wei, X.-X.; Qi, L.; Xu, G.-L.; Duan, J.; Liu, X.-Y.; Shu, X.-Z. J. Am. Chem. Soc. 2021, 143, 4536. 